\title{
Bandwidth Enhancement of Microstrip Patch Antenna Using Metamaterials
}

\author{
Ananya Parameswaran, Shri .Satya Bhushan Shukla, Shri. K K Mukundan, \\ Dr.T.Shanmuganantham. \\ M.Tech Electronics, Dept of Electronics Engg., Pondicherry University. \\ Sci/Engr 'SD',ADRFD/RFSG/AVN, VSSC, Thiruvananthapuram, India. \\ HEAD, ADRFD, VSSC, Thiruvananthapuram, India. \\ Assistant Professor, Dept. of Electronics Engg., Pondicherry University.
}

\begin{abstract}
In this paper, Bandwidth enhancement of a patch using a new kind of material, so called 'Metamaterial', is being discussed. This new material called Electromagnetic Band Gap (EBG) mushroom type structure is used to replace the commonly used ground plane for the simple patch. The simple patch showed a percentage bandwidth of $1.9 \%$. The bandwidth was found to increase greatly to $39.20 \%$ after the replacement of the ground plane. The patch antenna was simulated using the Computer Simulation Tool (CST) Microwave studio.
\end{abstract}

Keywords: Electromagnetic Band Gap material, Metamaterials, Microstrip patch antenna, Mushroom Structure, Surface waves

\section{Introduction:}

A microstrip patch antenna consists of a radiating patch on one side of a substrate and a ground plane on the other side of the substrate. The advantages of a patch antenna are: light weight and low volume, low fabrication cost, compatible with MIC technology and so on. Even though it has the above mentioned advantages, it suffers from the some drawbacks like low bandwidth , surface waves present in the substrate.

The major problem associated with planar antennas originates in the guiding of plane waves by a plane interface between two different media: conductor-dielectrics or dielectrics-dielectrics. The electromagnetic energy trapped between the interfaces, and forming into surface waves, is substantial: an elementary dipole, placed on a uniform substrate with no losses and represented by the relative dielectric constant $\varepsilon$, radiates $\varepsilon^{3 / 2}$ times more power into the substrate than into the air; a second problem is that the electromagnetic waves radiated into substrate and reaching the dielectric-air interface at angles greater than $\theta_{c}=\sin ^{-1}\left(\varepsilon^{-1 / 2}\right)$ are totally reflected. The power transferred into the surface waves does not contribute to the main radiation of the antenna, but it is scattered off the edges of the finite ground plane and leads to deep nulls and ripples in radiation patterns, increased back radiation, gain deterioration, lower polarization purity, etc. As a result, these surface waves degrade the antenna performance [1]. By using a new material called "Metamaterial" the surface waves can be removed .

The organisation of the paper is as follows. In part I, the basics of metamaterial is covered. In part II, the design of a simple patch fed by a microstrip line is explained using the basic design equations. Then the design steps for microstrip patch with mushroom ground plane is explained. Finally, the results are discussed in the results and discussion part.

\section{Background of Metamaterials}

Metamaterials are periodic structures consisting of sub wavelength metalo-dielectric scatterers having a periodicity that is much smaller than the impinging and guided wavelengths. These constituent scatterers or "inclusions" behave like "artificial molecules" that scatter an incident electromagnetic wave giving rise to macroscopic effective material parameters such as a permittivity, a permeability, and a refractive index [2].

The Electromagnetic Band Gap (EBG) structures are one kind of metamaterials which are capable of suppressing the surface waves within the substrate. Based on the geometry, the EBG are classified as 1-D, 2-D and 3-D, ie. One dimensional, Two dimensional and Three dimensional respectively. The 2-D EBG are further classified as mushroom type and the planar type. The basic difference between the mushroom and planar type is the presence or absence of a via. The figures of these are as shown below. 

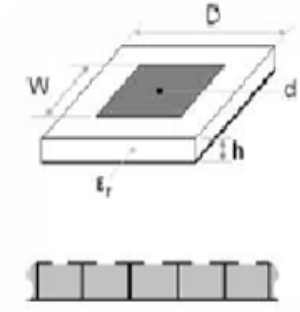

a)
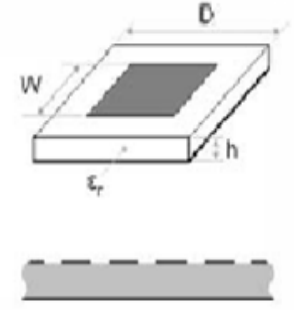

B)

Fig.1Mushroom and Planar EBG structures [8]

The mushroom type EBG consists of an array of square patches, each having a via at the centre which connects the square patch to the ground plane. The connection to the ground plane through the via acts as an inductance and the spacing between adjacent patches acts as a capacitance. Mushroom EBG with its inductance ' $\mathrm{L}$ ' and capacitance ' $\mathrm{C}$ ' behaves as a parallel resonant $\mathrm{LC}$ circuit. We know that a parallel resonant circuit has a very high impedance at its frequency of resonance. This is the reason why mushroom EBG is also called as 'high impedance surface'. Because of this high impedance, it does not allow an electromagnetic wave to pass through it, but reflects it in phase with the main radiated wave thereby improving the radiation characteristics of the patch in a way giving a better performance. Since, this structure provides a bandgap for the electromagnetic impinging on it, it is also called as Electromagnetic Band Gap (EBG)materials .[3]

\section{Design Of A Patch Antenna}

The design equations of a patch are as given below.[4]

The width ' $\mathrm{W}$ ' of the patch in $(\mathrm{mm})$

$$
W=\frac{c}{2 f_{o} \sqrt{\frac{\left(\varepsilon_{r}+1\right)}{2}}}
$$

The effective dielectric constant $\varepsilon_{\text {reff, }}$ is given by:

$$
\varepsilon_{\text {reff }}=\frac{\varepsilon_{r}+1}{2}+\frac{\varepsilon_{r}-1}{2}\left[1+12 \frac{h}{W}\right]^{-\frac{1}{2}}
$$

Where,

$\varepsilon_{\mathrm{r}}=$ relative permittivity of the substrate used.

$\mathrm{h}=$ height of the substrate

$\mathrm{w}=$ width of the patch

The effective length, $\mathrm{L}_{\text {eff }}$ of the patch is given by:

$$
L_{e f f}=\frac{c}{2 f_{o} \sqrt{\varepsilon_{r e f f}}}
$$

Where,

$\mathrm{f}_{0}=$ operating frequency of the patch

$\varepsilon_{\text {reff }}$ effective dielectric constant of the substrate

The differential increase in length, $\Delta \mathrm{L}$ is 


$$
\Delta L=0.412 h \frac{\left(\varepsilon_{\text {reff }}+0.3\right)\left(\frac{W}{h}+0.264\right)}{\left(\varepsilon_{\text {reff }}-0.258\right)\left(\frac{W}{h}+0.8\right)}
$$

The actual length of the patch is given as:

$$
L=L_{\epsilon f f}-2 \Delta L
$$

Using the above formulae, the dimensions of the patch are :

$\mathrm{W}=38 \mathrm{~mm}$

$\mathrm{L}=30 \mathrm{~mm}$

The simple patch is shown in Fig.2

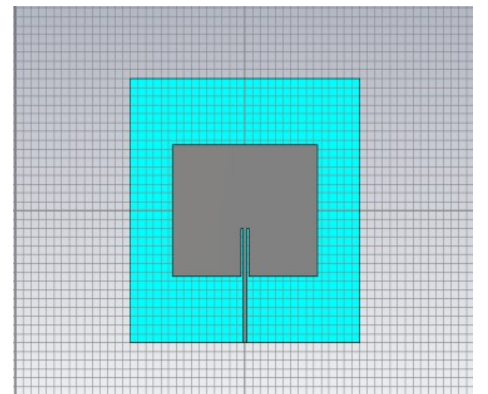

Fig.2 A simple microstrip fed patch antenna

The 3D pattern and return loss curve of the above patch are shown in Fig.3.

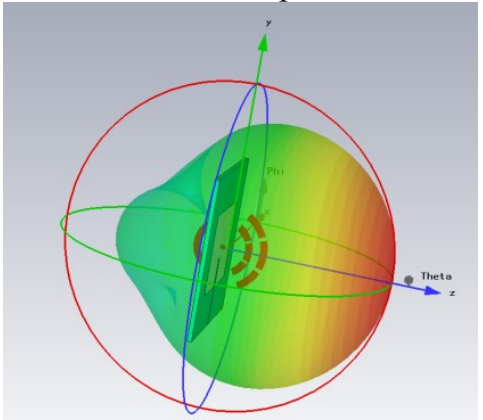

Fig.3a 3D radiation pattern

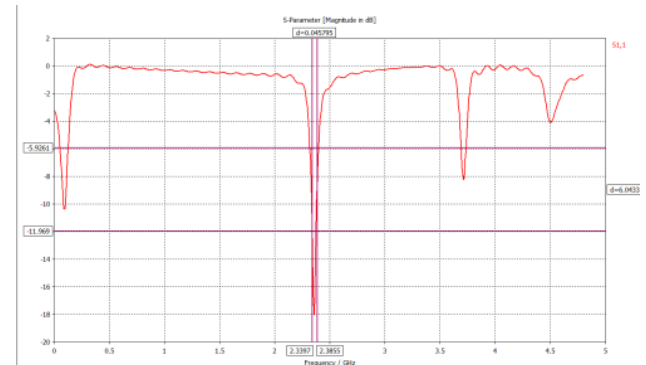

Fig.3b Bandwidth of simple microstrip line fed patch

From, Fig.3a, we observe that the microstrip patch has side and back lobes in addition to the main radiated beam. The presence of these lobes are attributed to edge refraction of surface waves which are present in the 
substrate. Also, from Fig3b,we find that, the microstrip patch has a very narrow bandwidth of $45.7 \mathrm{MHz}$, which when put in percentage, comes to be $1.9 \%$.

In this paper, a novel method is presented, which greatly increases the bandwidth of the patch.

\section{Design Of Mushroom EBG}

The important parameters of a mushroom structure are:

Width of the patch, ' $w$ '

Gap between adjacent patches, ' $\mathrm{g}$ '

Height of the substrate, ' $h$ '

Radius of the via, ' $r$ '

These parameters are given by:

$$
\begin{aligned}
& w=0.10 \lambda_{12} \\
& g=0.02 \lambda_{12} \\
& h=0.04 \lambda_{12} \\
& r=0.005 \lambda_{12}
\end{aligned}
$$

Here, $\lambda_{1}$ is the free space wavelength corresponding to the operating frequency of the patch and $\lambda_{2}$ is the wavelength of the wave considering the dielectric constants of both the substrates, viz, the substrate for the patch and that for the mushroom structure.

The values for the above parameters are: $\mathrm{w}=6 \mathrm{~mm}, \mathrm{~g}=4 \mathrm{~mm}, \mathrm{~h}=2.4 \mathrm{~mm}$ and $\mathrm{r}=0.35 \mathrm{~mm}$.

Fig.4 shows the mushroom EBG structure.

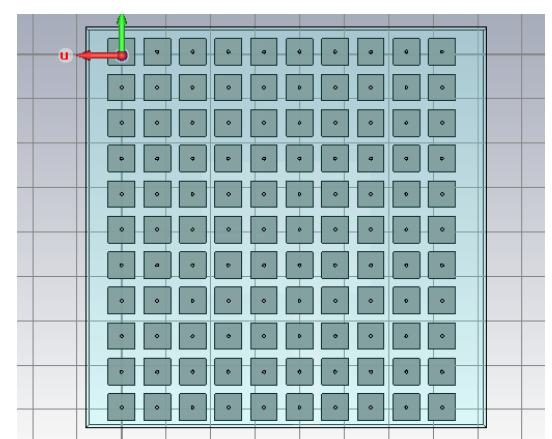

Fig.4 Mushroom EBG as ground plane for the patch.

As discussed in the background of Metamaterials, this structure behaves likes a parallel LC circuit. The values of ' $\mathrm{L}$ ' and 'C' are functions of the mushroom parameters as expressed below.

$L=\mu_{0} h\left\lfloor\ln \frac{4 h}{d}+0.5 \frac{d}{h}-0.75\right\rfloor$

$C=\frac{w \varepsilon_{0}\left\lfloor\varepsilon_{r 1}+\varepsilon_{r 2}\right\rfloor}{\pi} * \cosh ^{-1}\left[\frac{2 w+g}{g}\right]$

The relations for the resonant frequency and the bandwidth are as below:

$$
\begin{aligned}
& f_{r}=\frac{1}{2 \pi \sqrt{L C}} \\
& B W=\frac{1}{Z_{0}} * \sqrt{\frac{L}{C}}
\end{aligned}
$$


The bandwidth obtained using the structure shown in Fig, 4 is $31.78 \%$.

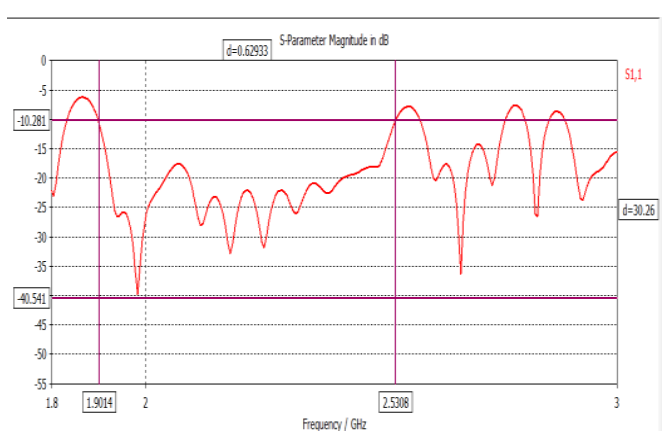

Fig.5 Bandwidth of patch after using simple square mushroom as the ground plane.

When, metal strips are introduced on the patch substrate, surrounding it, the $\%$ bandwidth was further found to be increased to $32.09 \%$.

The square mushroom was replaced with various other structures as shown below:

$6 a$

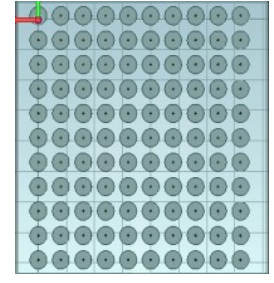

x

$x x \operatorname{xin} x \sin x=$

x

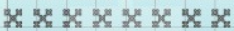

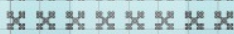

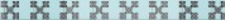

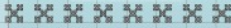

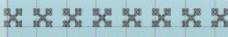

$6 \mathrm{~b}$

X 30

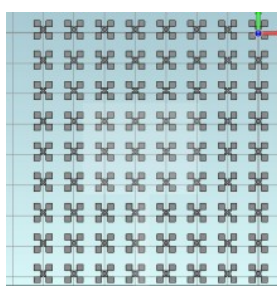

$6 c$

Fig .6 Different mushroom structures used.

(6a) circular EBG, (6b)Fractal minkowski , (6c) Fractal minkowski with $2^{\text {nd }}$ iteration.

The values of $\%$ bandwidth obtained using the above structures are tabulated below:

\begin{tabular}{|c|c|}
\hline TYPE OF STRUCTURE USED & $\begin{array}{c}\text { \%BANDWIDTH } \\
\text { OBTAINED }\end{array}$ \\
\hline SIMPLE RECTANGULAR PATCH & 1.90 \\
\hline CIRCULAR MUSHROOM & 29.16 \\
\hline SQUARE MUSHROOM & 31.78 \\
\hline $\begin{array}{c}\text { SQUARE MUSHROOM WITH METAL } \\
\text { STRIPS SURROUNDING THE PATCH [7] }\end{array}$ & 32.09 \\
\hline MINKOWSKI STRUCTURE & 36.60 \\
\hline $\begin{array}{c}\text { MINKOWSKI STRUCTURE WITH } \\
\text { METAL STRIPS SURROUNDING THE } \\
\text { PATCH }\end{array}$ & 37.68 \\
\hline $\begin{array}{c}\text { MINKOWSKI WITH 2 ITERATION AND } \\
\text { METAL STRIPS SURROUNDING PATCH }\end{array}$ & 39.20 \\
\hline
\end{tabular}




\section{Results And Discussion}

The simple patch was showing a bandwidth of $1.9 \%$. The reason for this very narrow bandwidth is given in the introduction part with respect to the presence of surface waves in the patch substrate.

The PEC ground plane for the patch was replaced with the square EBG mushroom structure. The great increase in the bandwidth is attributed to the presence of inductance and capacitance.

Then the fractal structure was used to replace the basic mushroom EBG. The indentations present in the minkowski structure act like inductance which adds with the inductance provided by the via. And hence, this results in the further improvement in the \% bandwidth.

\section{Conclusion}

In this paper, bandwidth enhancement of a simple microstrip patch antenna is discussed using a new material called, mushroom EBG metamaterial. The percentage bandwidth of the patch was improved greatly from $1.9 \%$ to $31.78 \%$ using square mushroom EBG structure as ground plane.Various structures were used to replace the basic mushroom for the ground plane of the patch. Then, finally a comparison was made between these various structures.

\section{References}

[1] Sandhya Bhavsar, Prof. Bharati Singh "Electromagnetic Band Gap Structures Incorporated In Antenna Array: A Review", International Journal of Computer Technology and Electronics Engineering (IJCTEE) Volume 3, Special Issue, March-April 2013,

[2] George V Eleftheraides \& Keith G Balmain,"Negative Refraction Metamaterials, Fundamental Principles and Applications .", Proceedings of the IEEE, Vol. 99, No. 10, October 2011

[3] Fan Yang and Yahya Rahmat- Samii, "Reflection Phase Characterizations of the EBG Ground Plane for Low Profile Wire Antenna Applications", IEEE TRANSACTIONS ON ANTENNAS AND PROPAGATION, VOL. 51, NO. 10, OCTOBER 2003.

[4] C. A. Balanis, "Antenna theory, Analysis and Design", $2^{\text {nd }}$ Edition.

[5] Raj Kumar and Jayashree P. Shinde, "On the Effect of Fractal Electromagnetic Band Gap on Antenna Performance", IETE JOURNAL OF RESEARCH, VOL 56, ISSUE 6 , NOV-DEC 2010.

[6] Tian Hong Loh, "High Impedance Surface Electromagnetic Band Gap Metamaterials:Design approach and applications for antenna engineering".

[7] Simon Tse, John Batchelor, Paul Young, Richard Langley, "Enhanced MEBG patch antenna performance by using a closed edge substrate".

[8] Peter Kovács, Zbyněk Raida, Marta Martínez-Vázquez, "Parametric Study of Mushroom-like and Planar Periodic Structures in Terms of Simultaneous AMC and EBG Properties", RADIOENGINEERING, VOL.17, NO.4, DECEMBER 2008. 